\title{
Primary Percutaneous Coronary Intervention Followed by Valve Surgery for Acute Coronary Syndrome at Left Main Trunk Complicated With Severe Aortic Stenosis
}

\author{
Akira ShIKUMA, ${ }^{1}$ MD, Jun ShIRAISHI, ${ }^{1}$ MD, Kazunari OKawa, ${ }^{2}$ MD, \\ Masaki Yashige, ${ }^{1}$ MD, Keisuke ShouI, ${ }^{1}$ MD, Daisuke Ito, ${ }^{1}$ MD, Masayoshi Kimura,,${ }^{1}$ MD, \\ Eigo Kishita, ${ }^{1}$ MD, Yusuke NaKagawa, ${ }^{1}$ MD, Masayuki Hyogo, ${ }^{1}$ MD, \\ Akiyuki TAKAHASHI, ${ }^{2} \mathrm{MD}$, and Takahisa SaWAdA, ${ }^{1} \mathrm{MD}$
}

\begin{abstract}
SUMMARY
An 89-year-old woman appeared to have acute coronary syndrome at the left main trunk (LMT) complicated with severe aortic stenosis, moderate-severe mitral regurgitation, depressed left ventricular (LV) function, and multivessel disease. Because of sustained hypotension even under intra-aortic balloon pumping support during emergency coronary angiograhy, we performed primary percutaneous coronary intervention solely for the LMT lesion using a bare metal stent, leading to recovery from the shock state. On the second hospital day, based on our heart-team consensus, we performed aortic valve replacement and coronary artery bypass grafting surgery, and added edge-to-edge repair (Alfieri stitch) of the mitral valve, resulting in complete revascularization and dramatically improved LV function. (Int Heart J 2017; 58: 125-130)
\end{abstract}

Key words: Acute myocardial infarction, Bare metal stent, Coronary artery bypass grafting, Alfieri stitch, Intra-aortic balloon pumping

$\mathrm{A}$ ccumulating evidence has shown that primary percutaneous coronary intervention (PCI) has great prognostic effects particularly in acute myocardial infarction (AMI) patients complicated with cardiogenic shock; $;^{1-4)}$ however, AMI at the left main trunk (LMT) culprit, frequently accompanied by cardiogenic shock, is a deteriorated condition with high in-hospital morbidity and mortality, and still remains a therapeutic challenge for cardiologists. ${ }^{5-9}$ On the other hand, surgical aortic valve replacement (AVR) combined with coronary artery bypass grafting (CABG) surgery has been an established therapeutic strategy for patients with severe aortic stenosis (AS) and coronary artery disease (CAD). We describe our heart-team experience with an unusual fatal case of acute coronary syndrome (ACS) at LMT complicated with severe AS and multivessel disease, in which hybrid primary PCI for the LMT culprit alone followed by AVR and concomitant CABG could be successfully performed, leading to complete revascularization and a hemodynamically stable condition.

\section{CASe Report}

An 89-year-old-female was admitted to our hospital with chest pain and nausea that had persisted for 2 hours. Sixteen months before she had already been diagnosed as having se- vere AS with a transvalvular peak pressure gradient (PG) of $141 \mathrm{mmHg}$, a mean PG of $77 \mathrm{mmHg}$, a calculated aortic valve area of $0.52 \mathrm{~cm}^{2}$, moderate-severe mitral regurgitation (MR), and no asynergy with an ejection fraction of 0.70 on transthoracic echocardiography; however, she did not agree to undergoing AVR. Her coronary risk factors included hypertension and dyslipidemia. On arrival, her blood pressure was 110/68 $\mathrm{mmHg}$ and her pulse rate was 100 beats/minute and regular. Her physical examination was unremarkable, except for a systolic murmur at the right sternal border in the third to the fourth intercostal space. An electrocardiogram (ECG) showed significant ST-segment depression in precordial leads, I, aVL, II, and aVF in addition to ST-segment elevation in aVR (Figure 1 ), and a chest radiograph revealed cardiomegaly with lung congestion (Figure 2A). Subsequent transthoracic echocardiography revealed a decreased motion in the apico-anteroseptal wall of the left ventricle concomitant with severe AS and moderate-severe MR. Routine blood testing showed slight elevation of cardiac enzymes (CPK 179 IU/L, CPK-MB 27 IU/L, troponin $0.19 \mathrm{ng} / \mathrm{mL}$ ) without leukocytosis, definite renal dysfunction, and anemia (white blood cell $4000 / \mu \mathrm{L}, \mathrm{Cr} 0.69 \mathrm{mg} /$ $\mathrm{dL}$, eGFR $59 \mathrm{~mL} /$ minute $/ 1.73 \mathrm{~m}^{2}$, hemoglobin $12.2 \mathrm{~g} / \mathrm{dL}$ ).

Based on the diagnosis of ACS complicated with severe AS, emergency coronary angiography (CAG) was performed via the left radial artery. Right CAG indicated significant sten-

From Departments of ${ }^{1}$ Cardiology and ${ }^{2}$ Cardiovascular Surgery, Kyoto First Red Cross Hospital, Kyoto, Japan.

Address for correspondence: Jun Shiraishi, MD, Department of Cardiology, Kyoto First Red Cross Hospital, 15-749 Honmachi, Higashiyama-ku, Kyoto 605-0981, Japan. E-mail: risa11221998@yahoo.co.jp

Received for publication April 17, 2016. Revised and accepted May 30, 2016.

Released in advance online on J-STAGE January 17, 2017.

All rights reserved by the International Heart Journal Association. 

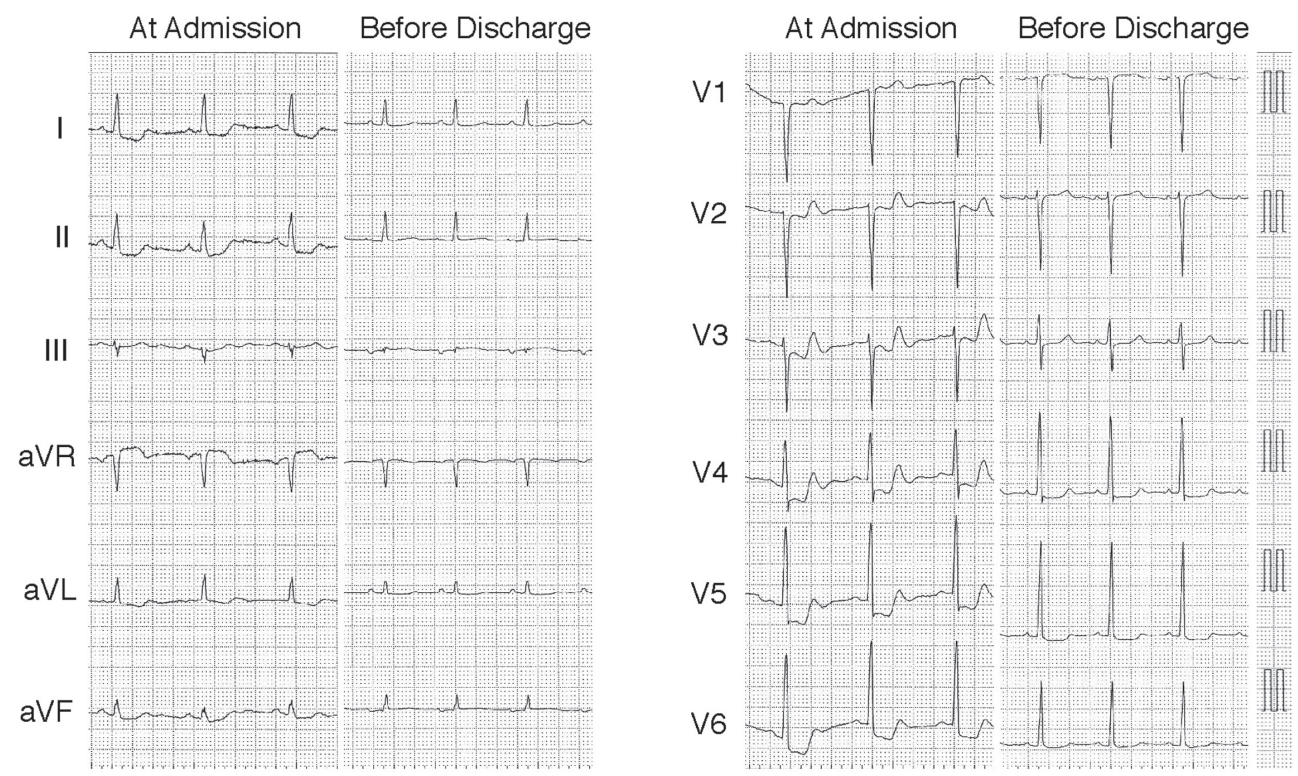

Figure 1. Electrocardiograms at admission and before discharge. Significant resolution of ST-segment change was observed before discharge.
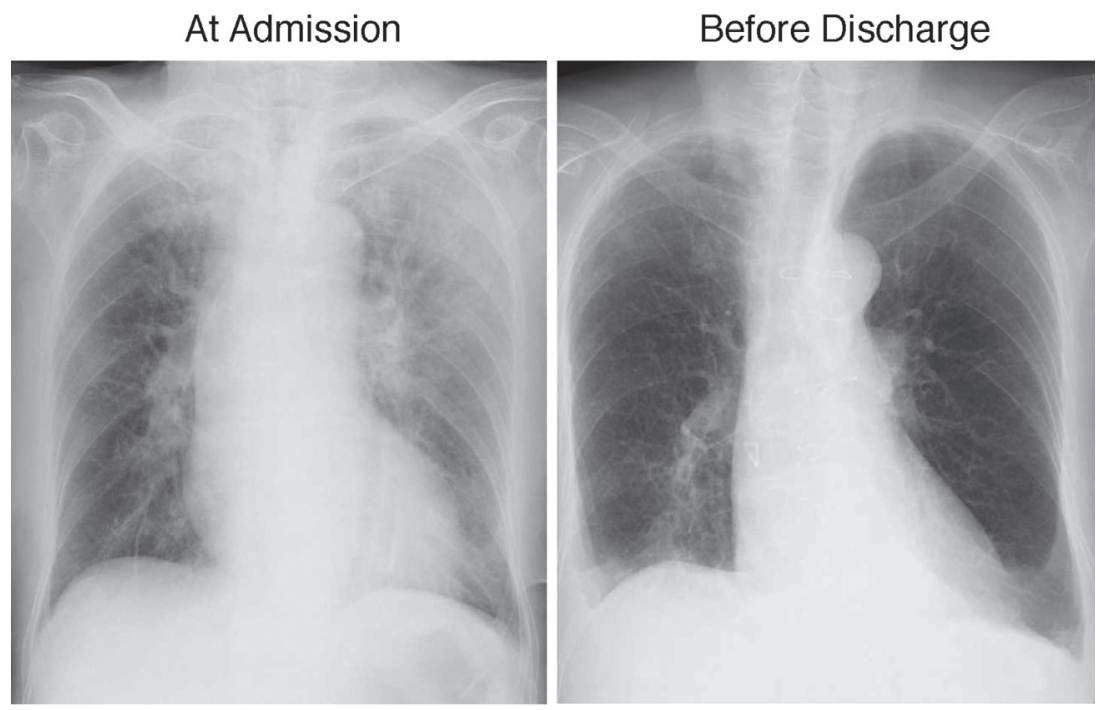

Figure 2. Chest radiographs at admission (supine position) and before discharge (standing position). No definite lung congestion was detected before discharge.

osis in the proximal right coronary artery (RCA) (Figure 3A). During the right CAG, systolic blood pressure decreased to 60 $70 \mathrm{mmHg}$ and then a shock state persisted. On fluoroscopy, in addition to a severely calcified aortic valve with poor mobility, marked calcification at LMT was observed (Figure 3A). Taking refractory hypotension and the possibility of LMT as the culprit into consideration, before left CAG, we immediately inserted an intra-aortic balloon pump (IABP) and a 7-Fr sheath into her right femoral artery and right femoral vein, respectively. The first shot on the left CAG revealed severe stenosis in the ostium-body segment of the LMT (Figure 3B). After we confirmed the LMT lesion as the ACS-culprit site, in consideration of forthcoming AVR, we planned to perform transradial primary PCI solely for the LMT from the ostium to the body if possible, using a short bare metal stent (BMS) under IABP support and catecholamine administration as well as under onsite surgery backup. After premedication with aspirin $200 \mathrm{mg}$ and prasugrel $10 \mathrm{mg}$ as well as intravenous administration of 7,000 U of unfractionated heparin, using a 7-Fr Judkins left short-tip type guiding catheter, a floppy guidewire was passed and advanced into the distal LAD without difficulty. Subsequent left CAG via the guiding catheter indicated mild stenosis in the distal LMT and intermediate diffuse stenosis in the proximal left anterior descending coronary artery (LAD) in addition to severe stenosis in the ostium-body segment of the LMT (Figure 3C), and another floppy guidewire was crossed 

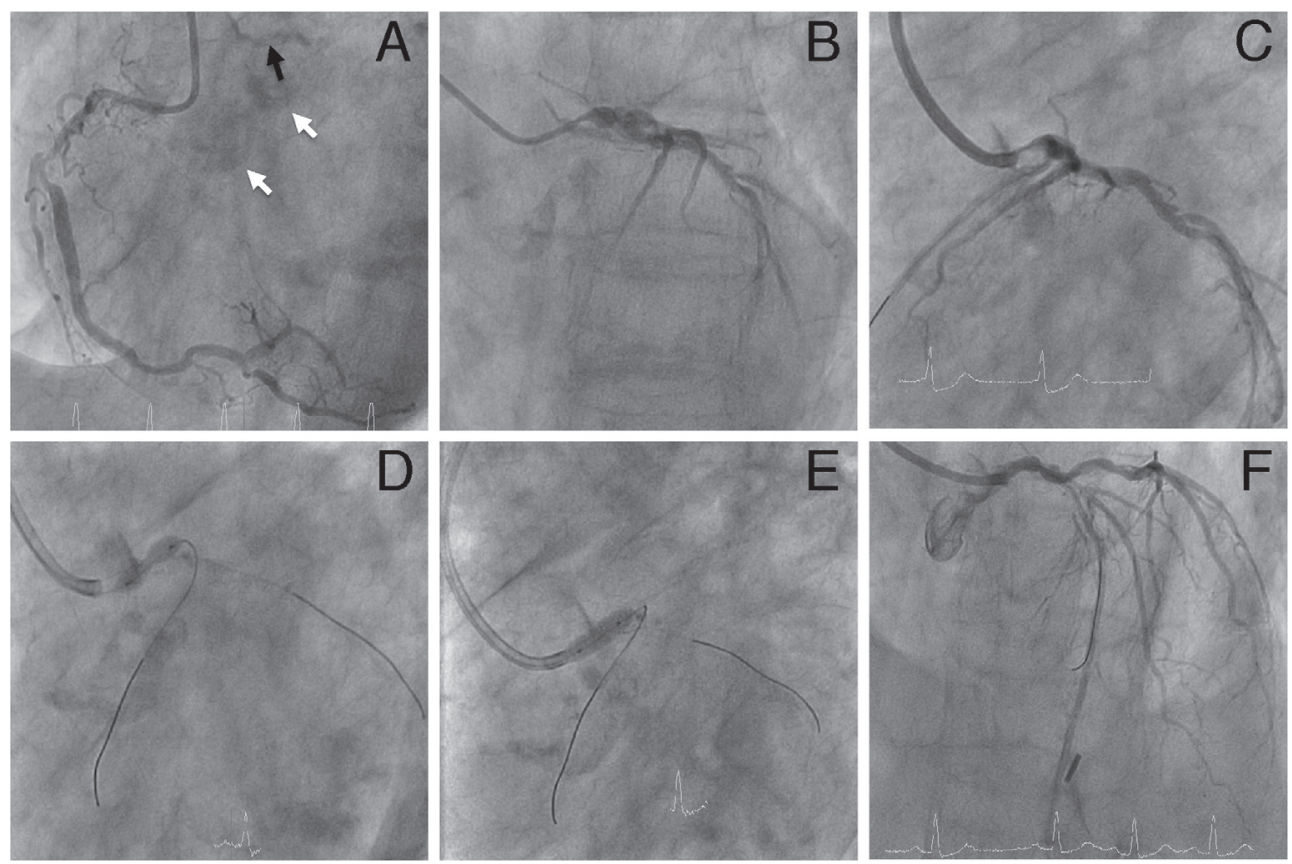

Figure 3. Emergency coronary angiography (CAG) and primary PCI for LMT lesion (C-E, left anterior view). A: Right CAG depicted significant stenosis in the proximal RCA (left anterior view). Severe calcifications at the aortic valve (white arrows) and at the LMT (black arrow) were suspected. Left CAG via angiography catheter (B, anterior-posterior view) and guiding catheter (C) showed severe stenosis in the ostium-body of LMT. D: Implantation of a bare metal stent in the ostiumbody of LMT with indentation. E: Postdilation with a $4.0 \mathrm{~mm}$ balloon without indentation. F: Final left CAG after PCI (left anterior cranial view) depicted residual mild stenosis in the distal LMT and intermediate diffuse stenosis in the proximal LAD.

into the distal left circumflex coronary artery (LCx). After predilation using a $2.25 \mathrm{~mm}$ balloon catheter and a $2.5 \mathrm{~mm}$ scoring balloon catheter, intravascular ultrasound imaging (IVUS) depicted almost circumferential calcification with a probable crack at the LMT lesion and diffuse plaque at the proximal LAD. Under the guidance of IVUS, a BMS (Liberte, 4.0/8 $\mathrm{mm}$, Boston Scientific) was implanted at $20 \mathrm{~atm}$ from the ostium to the body in the LMT (Figure 3D). Since stent underexpansion still remained on fluoroscopy and IVUS even after postdilation by a $3.5 \mathrm{~mm}$ balloon catheter at $24 \mathrm{~atm}$, the stent was subsequently postdilated with a $4.0 \mathrm{~mm}$ balloon catheter at $24 \mathrm{~atm}$ (Figure 3E) with an acceptable angiographic result (Figure 3F). Final IVUS showed complete stent apposition with an optimal placement at the ostial LMT. During the primary PCI after IABP insertion, systolic blood pressure of approximately $70 \mathrm{mmHg}$ and diastolic augmentation blood pressure of approximately $100 \mathrm{mmHg}$ had not changed much, even at the time of each balloon inflation within 10 seconds in the LMT, and neither malignant arrhythmia nor slow flow/no reflow phenomenon was observed. Right cardiac catheterization immediately after the primary PCI showed a cardiac output of $3.10 \mathrm{~L} /$ minute, a cardiac index $(\mathrm{CI})$ of $2.30 \mathrm{~L} /$ minute $/ \mathrm{m}^{2}$, and mean pulmonary capillary wedge pressure of $25 \mathrm{mmHg}$ under support of IABP, catecholamine infusion, and biphasic positive airway pressure (BIPAP).

After the primary PCI, the degree of ST-segment change on ECG ameliorated, her systolic blood pressure gradually increased to $100-110 \mathrm{mmHg}$, and the dose of catecholamine infusion was gradually decreased. Although her hemodynamics as well as oxygenation remained almost unchanged under IABP, a small amount of catecholamine infusion, and BIPAP support, the value of CPK increased up to $2994 \mathrm{IU} / \mathrm{L}$ on the second hospital day. Based on the clinical course and on the angiographic findings, our heart team, which consisted of interventional cardiologists and cardiovascular surgeons, discussed and concluded that immediate AVR concomitant with CABG for LAD and RCA was indispensable to overcome her deteriorated condition, while double valve surgical intervention for both severe AS and functional MR might have high operative risk because of her super-high age and post-ACS condition.

On the second hospital day, our cardiovascular surgery team therefore performed AVR and CABG surgery (left internal thoracic artery-LAD, aorta-saphenous vein graft-posterior descending branch). The calcified tricuspid aortic valve and proximal edge of the BMS implanted in the LMT were observed (Figure 4A). The AVR was performed using a bioprosthetic valve (Carpentar-Edwards PERIMOUNT MAGNA EASE $19 \mathrm{~mm}$ ). During weaning from cardiopulmonary bypass, a decrease of blood pressure and elevation of pulmonary artery pressure were observed. The surgery team speculated that difficulty in weaning the cardiopulmonary bypass might have been due to the presence of severe MR on transesophageal echocardiography so edge-to-edge repair between the A1A2 border segment and P1P2 border segment (Alfieri stitch) of the mitral valve (Figure 4B) was performed. After the mitral valve repair, the MR significantly decreased to the mild level, and the patient was successfully weaned from the by- 

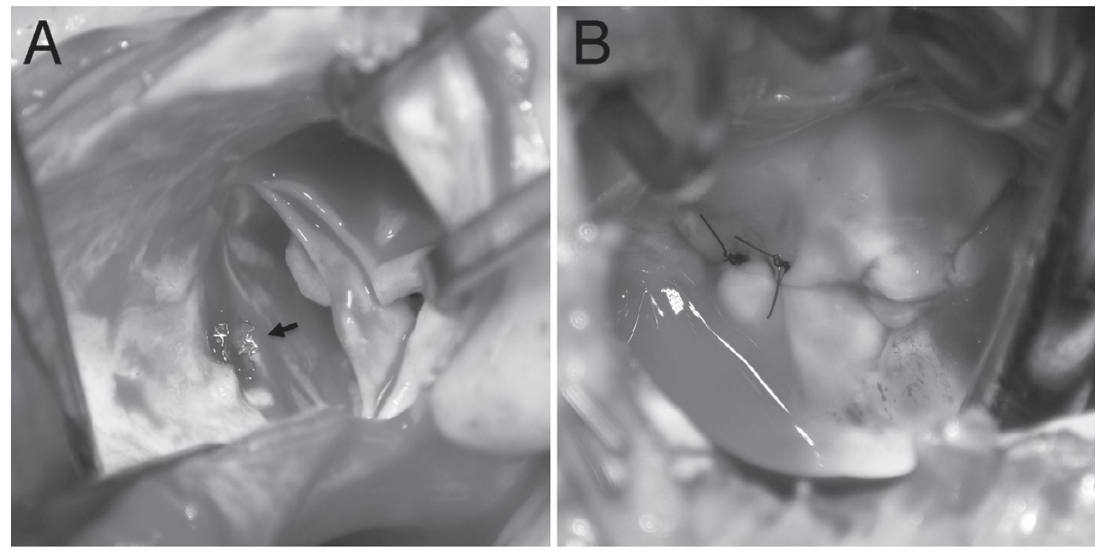

Figure 4. A: Intraoperative photograph showing calcified aortic valve and proximal edge of the bare metal stent implanted in the LMT (black arrow). B: Intraoperative photograph showing edge-to-edge repaired mitral valve (Alfieri stitch).

pass. After surgery, her condition gradually improved under anticoagulation therapy using intravenous infusion of unfractionated heparin without antiplatelet agents, and weaning from the IABP and the respirator was accomplished on the 6th hospital day and the 7th hospital day, respectively. We started the dual antiplatelet therapy (DAPT) (aspirin $100 \mathrm{mg} /$ day and clopidogrel $75 \mathrm{mg} /$ day) again on the 6th hospital day. Four units of red cell concentrates and 15 units of platelet concentrates were transfused on the 6th hospital day (the 5th postoperative day) because of postoperative anemia and thrombocytopenia concomitant with sustained drainage from a chest tube. Since then no blood transfusions were performed even after readministration of antiplatelet agents. Since paroxysmal atrial fibrillation and mild mitral stenosis with a mitral valve area (MVA) of $1.80 \mathrm{~cm}^{2}$ (pressure half time method) due to the mitral valve repair were detected on the 4 th postoperative day, we exchanged the heparin infusion for warfarin and kept the anticoagulation therapy. Next, we exchanged the triple therapy (DAPT plus warfarin) with the combination of clopidogrel plus warfarin on the 20th hospital day. Echocardiography 2 weeks after the surgery revealed no apparent asynergy with an ejection fraction of 0.77 and mitral stenosis with MVA of 1.41 $\mathrm{cm}^{2}$. On the 23rd hospital day, post-CABG angiography showed optimal patency of the grafts and mild focal narrowing at the implanted BMS in the LMT (Figure 5A-5D). Before discharge, ECG revealed only mild ST-segment depression in V4-6 leads (Figure 1), and a chest radiograph showed no definite lung congestion (Figure 2B). After rehabilitation, the patient was discharged on the 45th hospital day and remains free of cardiac events.

\section{Discussion}

Coexistence of CAD is frequently observed in patients with AS, and surgical AVR with concomitant CABG surgery has been a first-line therapeutic strategy for patients with symptomatic severe AS and stable CAD. In contrast, PCI has been occasionally performed in severe-AS/CAD patients with contraindications or high risk for surgery, in those with ACS, and in those whose symptoms are judged by the attending physicians to be attributable mainly to CAD rather than AS. Re- cently, Goel, et al reported a 30-day mortality rate of $4.3 \%$ after PCI in patients with severe AS, which was high but not significantly different from that in propensity-matched patients without $\mathrm{AS}{ }^{10)}$ In this report, both groups had higher frequencies of ACS (approximately 60\%), diabetes, and renal failure as well as low ejection fraction (approximately 0.45). High prevalence of ACS status and these comorbid medical conditions but not severe AS itself might therefore account for the high 30-day mortality rate in the patients with severe AS. Moreover, the prevalence of PCI-related complications did not differ significantly between the 2 groups. Thus, in terms of short-term outcome, PCI might be a feasible revascularization therapy of choice among patients with severe AS not only in ACS condition like the present case but also in conditions in which AVR/CABG is considered to have contraindications or more procedure-related risk.

The existence of CAD has been reported to increase the procedure-related risk of surgical AVR, ${ }^{11)}$ while surgical intervention consisting of AVR and CABG has a higher post-operative mortality than AVR alone. ${ }^{12,13)}$ A hybrid procedure with PCI followed by valve surgery has been therefore considered to be one of the therapeutic options among patients with valve disease and concomitant $\mathrm{CAD},{ }^{14-16)}$ and particularly for those presenting with ACS like the present case, this hybrid approach is an inevitable therapeutic strategy; however, excessive blood transfusion due to antiplatelet agent usage has been proposed as a crucial problem for the hybrid approach. According to a previous report that focused exclusively on patients with severe AS and CAD undergoing PCI on either the same day or the evening prior to minimally invasive AVR, all 18 patients received a drug-eluting stent (DES) under DAPT (aspirin before stenting and thereafter, clopidogrel loading just after stenting and thereafter), and had no reoperations for bleeding complications. ${ }^{15)}$ In addition, 8 of the 18 patients needed a postoperative blood transfusion, one patient died postoperatively from a colonic perforation, and there were no deaths during the mean follow-up of 19 months. ${ }^{15)}$ These results might be acceptable; however, there is a fortunate possibility that the DAPT did not confer a sufficient antiplatelet effect at the time of surgical AVR because of the short time interval between PCI and surgical AVR. In the present case, taking her super-high age and small appearance into consideration, we reduced the loading 

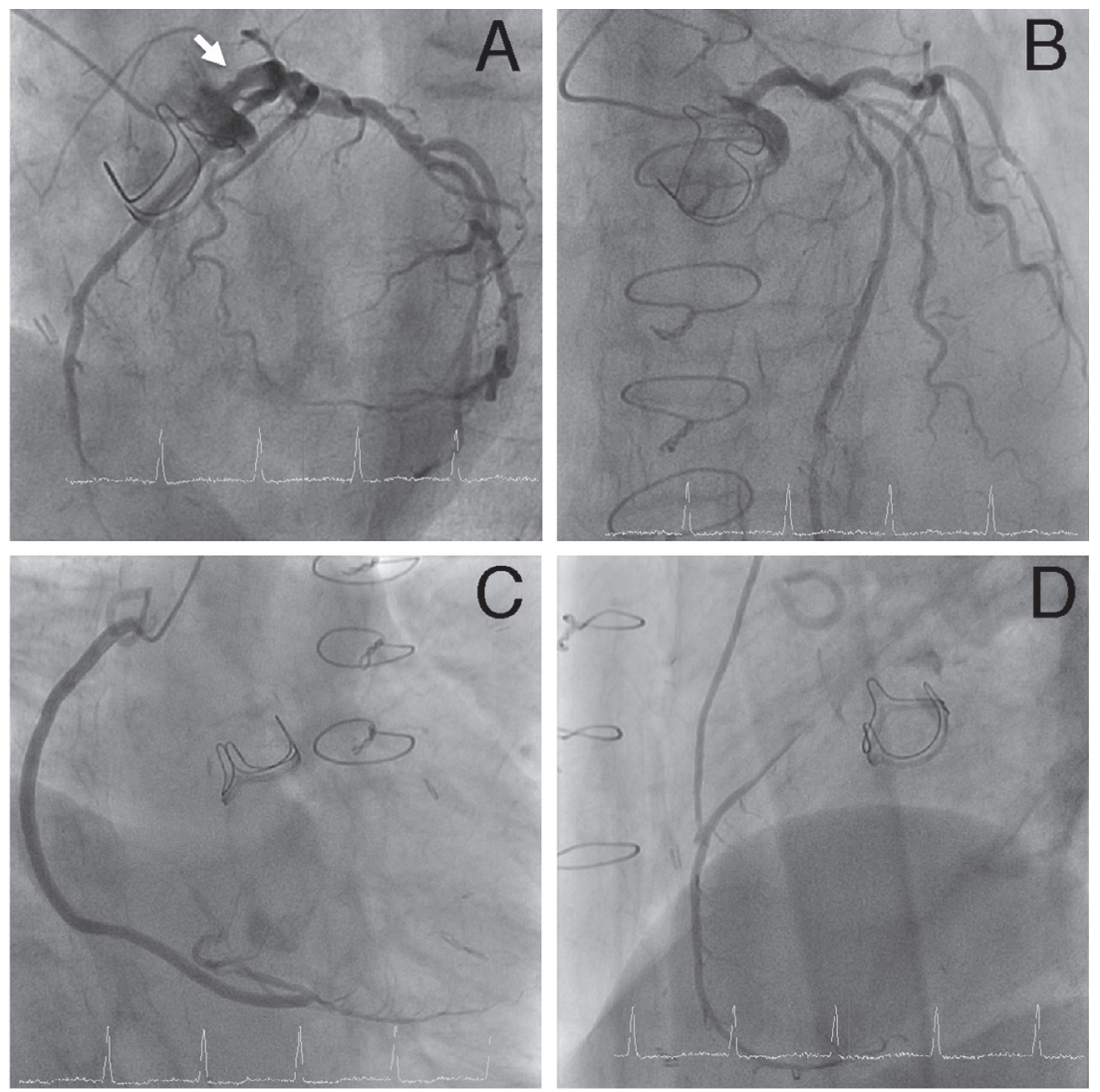

Figure 5. Postoperative coronary angiography (CAG) and graft angiography. A, B: Left CAG delineated the dilated LMT with focal mild narrowing (white arrow) (A, left anterior view; B, left anterior cranial view). C, D: Graft angiography confirmed patency of both grafts (C, aorta-saphenous vein graft-posterior descending branch; $\mathbf{D}$, left internal thoracic arteryLAD).

dose of prasugrel as the premedication for primary PCI. Nevertheless, it was difficult to do hemostasis during the surgery on the next day following the PCI, and transfusions of red cell concentrates and platelet concentrates were required on the 6th hospital day.

Another recent study compared 65 consecutive patients undergoing a hybrid approach combining staged PCI and minimally invasive valve surgery (AVR alone 31, mitral valve [MV] repair alone 14, MV replacement alone 10, AVR plus MV repair, or MV replacement 10) with 52 matched control patients undergoing conventional valve surgery plus CABG. ${ }^{16)}$ In the hybrid group, the median time interval between PCI and valve surgery was 24 days (interquartile range, 2.5-37 days), and at the time of surgery, 45 patients continued antiplatelet agents (DAPT 23, clopidogrel alone 18, aspirin alone 4), whereas 20 patients stopped antiplatelet agents 5 days before the valve surgery. There were no in-hospital deaths in the hybrid group compared with $2(3.8 \%)$ in the matched control group $(P=0.11)$. The hybrid group had a lower prevalence of the combined endpoint of 30-day death, renal failure, or stroke than the matched control group (1.5\% versus $28.8 \%, P=$ 0.001 ), while length of ICU stay and total hospital stay as well as average amount of blood transfusions did not vary significantly between the 2 groups. For the patients with a clinically stable condition, a longer time interval between PCI and surgical AVR might enable us to temporarily discontinue or weaken antiplatelet therapy before the surgery. In contrast, for the unstable patients with hemodynamic compromise like the present case, a shorter time interval (even the same day) in which antiplatelet agents could not exert an adequate antiplatelet effect at the time of surgical AVR, might be preferable. Taken together, in addition to hemodynamic status, specific clinical background, such as type of stent (DES or BMS), location of stent implantation, presence/absence of residual stenosis, and usage of antiplatelet/anticoagulant agents, should be taken into consideration to optimize the time interval between PCI and surgical AVR in each patient. An appropriate time interval between percutaneous and surgical interventions as well as a proper antiplatelet medication regimen should be examined in detail among the patients undergoing this hybrid therapy.

Multivessel PCI and DES usage during the primary PCI might not be suitable for the present case. Since there was a diffuse calcified lesion from the distal LMT to the proximal LAD in addition to severe stenosis in the ostium-body segment of the LMT (culprit lesion) and in the proximal RCA, it was risky to perform complex multivessel PCI in the shock state requiring IABP support and catecholamine administration. Moreover, DES usage was required for such complex multivessel PCI. During the emergency CAG/primary PCI under IABP support and catecholamine administration, we had estimated that AVR with or without CABG and mitral valve surgery should be performed at least within a few days to over- 
come her hemodynamic instability, and there was a possibility that antiplatelet agents might be unavailable for a while after the valve surgery. In addition, although the duration of DAPT administration has become shorter in patients with new-generation DES implantation, in the condition without antiplatelet therapy BMS might be even safer than new-generation DES regarding the prevention of stent-thrombosis particularly within a month after stent implantation. Taken together, a short BMS implantation in the LMT alone during the primary PCI followed by surgical AVR with CABG might be safer than complex multivessel PCI using DES during the primary PCI followed by surgical AVR alone. On the other hand, complex multivessel PCI using DES during the primary PCI followed by transcatheter aortic valve implantation (TAVI) alone could be an alternative therapeutic strategy of choice for super-highaged patients like the present case in a TAVI-capable hospital, while bleeding events have been reported to be common and related to worse prognosis even after TAVI. ${ }^{17)}$

In the critical ACS condition suspected of having LMT as the culprit vessel in the presence of diseased RCA and severe AS like the present case, it is preferable to insert an IABP before CAG or at least before engaging a catheter for LCA angiography in order to stabilize hemodynamics and not to exacerbate whole heart ischemia. Although the role of IABP usage in patients with severe AS remains uncertain, according to the previous report regarding patients with severe AS (transvalvular peak pressure gradient of $67 \pm 26.8 \mathrm{mmHg}$, a mean pressure gradient of $39.8 \pm 16.8 \mathrm{mmHg}$ ) in cardiogenic shock, the insertion of an IABP improved CI from $1.77 \mathrm{~L} / \mathrm{minute}^{2} \mathrm{~m}^{2}$ to 2.18 and $2.36 \mathrm{~L} /$ minute $/ \mathrm{m}^{2}$ at 6 and 24 hours, respectively, reduced systemic vascular resistance, and decreased central venous pressure. ${ }^{18)}$ In the present case, immediate IABP utilization before the LCA angiography might greatly contribute not only to successful completion of the emergency CAG/subsequent primary PCI but also to a stable clinical course during/ after the surgical intervention. Additionally, the absence of renal dysfunction and anemia at admission might account in part for her better in-hospital prognosis after AMI, although superhigh-aged women with AMI have a worse prognosis in general. $^{19)}$

Conclusion: The present case suggests that IABP-supported primary PCI for the LMT followed by surgical AVR and concomitant CABG under heart-team consensus could be a hybrid therapy of choice among high-risk patients with ACS at LMT complicated with severe AS and multivessel disease.

\section{Disclosure}

Conflict of interest: The authors have no conflicts of interest regarding the content of the manuscript.

\section{REFERENCES}

1. Moosvi AR, Khaja F, Villanueva L, Gheorghiade M, Douthat L, Goldstein S. Early revascularization improves survival in cardiogenic shock complicating acute myocardial infarction. J Am Coll Cardiol 1992; 19: 907-14.

2. Antoniucci D, Valenti R, Santoro GM, et al. Systematic direct angioplasty and stent-supported direct angioplasty therapy for car- diogenic shock complicating acute myocardial infarction: in-hospital and long-term survival. J Am Coll Cardiol 1998; 31: 294300 .

3. Hochman JS, Sleeper LA, Webb JG, et al. Early revascularization in acute myocardial infarction complicated by cardiogenic shock. SHOCK Investigators. Should We Emergently Revascularize Occluded Coronaries for Cardiogenic Shock. N Engl J Med 1999; 341: 625-34.

4. Dauerman HL, Goldberg RJ, White K, et al; GRACE Investigators. Revascularization, stenting, and outcomes of patients with acute myocardial infarction complicated by cardiogenic shock. Am J Cardiol 2002; 90: 838-42.

5. Pappalardo A, Mamas MA, Imola F, et al. Percutaneous coronary intervention of unprotected left main coronary artery disease as culprit lesion in patients with acute myocardial infarction. JACC Cardiovasc Interv 2011; 4: 618-26.

6. Sakai K, Nakagawa Y, Kimura T, et al. Primary angioplasty of unprotected left main coronary artery for acute anterolateral myocardial infarction. J Invasive Cardiol 2004; 16: 621-5.

7. Izumikawa T, Sakamoto S, Takeshita S, Takahashi A, Saito S. Outcomes of primary percutaneous coronary intervention for acute myocardial infarction with unprotected left main coronary artery occlusion. Catheter Cardiovasc Interv 2012; 79: 1111-6.

8. Yip HK, Wu CJ, Chen MC, et al. Effect of primary angioplasty on total or subtotal left main occlusion: analysis of incidence, clinical features, outcomes, and prognostic determinants. Chest 2001; 120: 1212-7.

9. Neri R, Migliorini A, Moschi G, Valenti R, Dovellini EV, Antoniucci D. Percutaneous reperfusion of left main coronary disease complicated by acute myocardial infarction. Catheter Cardiovasc Interv 2002; 56: 31-4.

10. Goel SS, Agarwal S, Tuzcu EM, et al. Percutaneous coronary intervention in patients with severe aortic stenosis: implications for transcatheter aortic valve replacement. Circulation 2012; 125 : 1005-13.

11. Aranki SF, Rizzo RJ, Couper GS, et al. Aortic valve replacement in the elderly. Effect of gender and coronary artery disease on operative mortality. Circulation 1993; 88: III17-23.

12. Tjang YS, van Hees Y, Körfer R, Grobbee DE, van der Heijden GJ. Predictors of mortality after aortic valve replacement. Eur J Cardiothorac Surg 2007; 32: 469-74. (Review)

13. Hannan EL, Wu C, Bennett EV, et al. Risk index for predicting inhospital mortality for cardiac valve surgery. Ann Thorac Surg 2007; 83: 921-9.

14. Byrne JG, Leacche M, Unic D, et al. Staged initial percutaneous coronary intervention followed by valve surgery ("hybrid approach") for patients with complex coronary and valve disease. J Am Coll Cardiol 2005; 45: 14-8.

15. Brinster DR, Byrne M, Rogers CD, et al. Effectiveness of same day percutaneous coronary intervention followed by minimally invasive aortic valve replacement for aortic stenosis and moderate coronary disease ("hybrid approach"). Am J Cardiol 2006; 98 : 1501-3.

16. Santana O, Funk M, Zamora C, Escolar E, Lamas GA, Lamelas J. Staged percutaneous coronary intervention and minimally invasive valve surgery: results of a hybrid approach to concomitant coronary and valvular disease. J Thorac Cardiovasc Surg 2012; 144: 634-9.

17. Kochman J, Rymuza B, Huczek Z, et al. Incidence, predictors and impact of severe periprocedural bleeding according to VARC-2 criteria on 1-year clinical outcomes in patients after transcatheter aortic valve implantation. Int Heart J 2016; 57: 35-40.

18. Aksoy O, Yousefzai R, Singh D, et al. Cardiogenic shock in the setting of severe aortic stenosis: role of intra-aortic balloon pump support. Heart 2011; 97: 838-43.

19. Shiraishi J, Kohno Y, Nakamura T, et al; AMI-Kyoto Multi-Center Risk Study Group. Prognostic impact of chronic kidney disease and anemia at admission on in-hospital outcomes after primary percutaneous coronary intervention for acute myocardial infarction. Int Heart J 2014; 55: 301-6. 